\title{
Polyamidoamine dendrimers as gene delivery carriers in the inner ear: How to improve transfection efficiency (Review)
}

\author{
HUI WANG, HAI-BO SHI and SHAN-KAI YIN \\ Department of Otolaryngology, Affiliated Sixth People's Hospital of Shanghai Jiao Tong University, \\ Otolaryngology Institute of Shanghai Jiao Tong University, Shanghai 200233, P.R. China
}

Received May 4, 2011; Accepted June 10, 2011

DOI: $10.3892 /$ etm.2011.296

\begin{abstract}
Hair cells in the cochlea can be damaged by various insults, including noise, drugs, infections and presbycusis, which may cause sensorineural hearing loss. Gene therapy is a novel therapeutic technology that, recently, has led to the idea of treating inner ear diseases on a genetic level. Depending on their characteristics, such as a high efficiency in delivery, the capability of specific targeting, multifunctionality, biodegradability, non-toxicity, non-immunogenicity and the capability of limiting DNA degradation, nanovectors, such as polyamidoamine (PAMAM) dendrimers for cellular gene delivery, provide a promising approach to eradicate genetic diseases. They are a new class of highly branched spherical polymers that are highly soluble in aqueous solution. Their unique surface is composed of positively charged primary amine groups which allow them to form stable complexes with plasmid DNA, oligonucleotides, antibodies and drugs. This review provides an overview of the characteristics of PAMAMs which may be used in gene transfer into the cochlea as well as the efforts to improve their transfection efficiency as gene-delivery carriers.
\end{abstract}

\section{Contents}

1. Introduction

2. Gene therapy in the inner ear with nanoparticle vectors

3. Characteristics of PAMAM dendrimers

4. Transfection activity of PAMAM dendrimers

5. Endeavors to enhance transfection efficiency

Correspondence to: Dr Shan-Kai Yin or Dr Hai-Bo Shi, Department of Otolaryngology, Affiliated Sixth People's Hospital of Shanghai Jiao Tong University, Otolaryngology Institute of Shanghai Jiao Tong University, 600 Yishan Road, Shanghai 200233, P.R. China

E-mail: yinshankai@china.com; haibo99@hotmail.com

Key words: polyamidoamine dendrimers, SuperFect, inner ear, gene therapy, nanoparticles

\section{Introduction}

Diseases of the inner ear, such as presbycusis, tinnitus, sudden hearing loss and vertigo, affect many individuals. However, no specific targeted therapeutic options are currently available. Gene therapy is expected to be effective in both the protection and the treatment of these exogenous and endogenous sources of inner ear diseases. The inner ear anatomically possesses several unique advantages as a model for gene therapy (1). The relative isolation of the cochlear compartments minimizes unwanted effects of the introduced gene into other tissues. Additionally, many physiological measurement tools, including cochlear microphonics, otoacoustic emissions, compound action potentials, endocochlear potentials and the auditory brain stem response, have been developed to monitor the function of specific cells in the inner ear and to assess the efficacy and safety of gene therapy. Many genes implicated in syndromic deafness have been recently cloned in the mouse and human to correct deafness. Several possibilities exist for using gene therapy in the cochlea to deal with deafness, such as the use of neurotrophin, which has been investigated in the protection of adult inner ear sensory cells against ototoxic chemicals and noise-induced damage.

\section{Gene therapy in the inner ear with nanoparticle vectors}

Gene therapy today is a novel therapeutic technology and has led to the idea of treating inner ear diseases on a genetic level. Depending on the characteristics, such as a high efficiency in delivery, the capability of specific targeting, multifunctionality, biodegradability, non-toxicity, non-immunogenicity and the capability of limiting DNA degradation, nanovectors in cellular gene delivery provide a promising approach to eradicate genetic diseases. Many investigators have explored the distribution of nanoparticles, such as polylactic/glycolic acid-encapsulated iron oxide nanoparticles (PLGA-NPs) $(2,3)$, lipid nanocapsules (4) or silica nanoparticles (5) in cochleae after application on the round window membrane. They were found in multiple locations in the organ of Corti and no hearing loss or inflammation was noted in the treated cochlea. Sun et al $(6,7)$ reported that hydroxyapatite nanoparticles efficiently mediated NT3 gene transfection both in vitro and in the cochlea of a living animal. Nerve growth factor-derived peptide functionalized nanoparticles were successfully transferred into the target cells of the 
inner ear, including spiral ganglion neurons, Schwann cells and nerve fibers (8). As a non-viral transfection agent, the polyamidoamine (PAMAM) dendrimer was reported to successfully introduce a reporter gene in cells of the inner ear both in vitro and in vivo (9). PAMAM dendrimers have been developed as the most promising gene-carrier candidates because of their well-defined structure, ease of controlling surface functionality and relatively high gene-transfection efficiency. Many studies have been performed in an attempt to produce efficient gene carriers using PAMAM dendrimers as base materials (10-12). This review discusses the characteristics of PAMAMs used in gene transfer into the cochlea and efforts to improve their transfection efficiency as gene-delivery carriers.

\section{Characteristics of PAMAM dendrimers}

In 1985, Tomalia et al (13) first synthesized PAMAM dendrimers containing both tertiary amines at branch points as well as primary amines at the termini. The sufficient surface amine groups enable them to interact with DNA, form complexes through their charge-based interactions and transfer DNA to cells efficiently both in vitro and in vivo. Recently, several biophysical measuring methods, including nuclear magnetic resonance, ultimate analysis and chromatograph analysis, have been utilized to investigate the structure and nature of PAMAM dendrimers. It was demonstrated that PAMAM dendrimers appear to be ideal gene vectors due to their physicochemical properties (13), including i) a high positive charge at physiological $\mathrm{pH}$ which facilitates electrostatic interaction with DNA to form complexes, ii) the presence of primary amine groups that are titratable in the endosome and a highly flexible structure that can collapse and swell (14), iii) the presence of an internal cavity surface shell to encapsulate guest molecules in the macromolecule interior (15) and iv) fast biodegradation which lowers cytotoxicity. PAMAM dendrimers have been synthesized in large quantities. The major structural difference in PAMAM dendrimers is related to their core molecule, which initiates the stepwise polymerization process. With each new generation from G0 to G10, the molecular weights and the numbers of active surface units of PAMAM dendrimers have increased exponentially, while their diameters have increased linearly. The toxicity is related to the generation and increases with the number of surface groups $(16,17)$ as the size influences the cytotoxicity of the dendrimer. The increasing branch density with generation is believed to have striking effects on the structure of dendrimers which are characterized by the presence of internal cavities to encapsulate a large number of reactive guest molecules. In recent years, in order to increase their targeting transfection efficiency, interactions of dendrimers with lipid bilayers, DNA and other molecules have been explored using theoretical and computational modeling methods (18-20). Hence, PAMAM dendrimers with high transfection efficiencies and minimal cytotoxicity may be effective in gene transfer and may have appropriate applications in various fields of biology and medicine.

\section{Transfection activity of PAMAM dendrimers}

Under physiological conditions, PAMAM dendrimer-DNA complexes have a positive net charge and bind to negatively charged surface molecules on cell membranes. Dendrimers are taken up into cells by non-specific endocytosis, and are then degraded by lysosomes. The targeting genes are then released and enter the nucleus to play a role in gene therapy. A transfection reagent called SuperFect ${ }^{\mathrm{TM}}$ consisting of activated dendrimers is commercially available (21). Transfection efficiency mediated by PAMAM dendrimers appears to be dependent on dendrimer generation, with larger sizes providing higher efficiency, as well as on the charge ratio of the complexes, in which a net positive charge is optimal and inversely related to their hydrophobicity (22).

In addition to the simple complexation of DNA with intact dendrimers, further enhancement of transfection utilizing either fractured (hydrolysis-degraded) dendrimers or the addition of cationic excipients, such as DEAE-dextran, has also been reported. Haensler and Szoka (23) first reported that when using luciferase and $\beta$-galactosidase-containing plasmids, dendrimers mediated a high-efficiency transfection in a variety of suspensions and adherent cultured mammalian cells. Dendrimer-mediated transfection was a function related to both the dendrimer/DNA ratio and the diameter of the dendrimer. Kukowska-Latallo et al (24) synthesized 20 different types of PAMAM dendrimers, and the efficiency of plasmid DNA transfection was examined by firefly luciferase and bacterial $\beta$-galactosidase. They found that the capability of a dendrimer to transfect cells appeared to depend on the size, shape and number of primary amino groups on the surface of the polymer. Bielinska et al (25) explored the mechanisms involved in the binding of PAMAM dendrimers to DNA and the nature of the DNA complexes that resulted from this interaction. Under electron microscopy, they found that a majority of the plasmid DNAs were contracted into isolated toroids, and also revealed larger, more irregular aggregates of the polymer and DNA. The binding of plasmid DNA to dendrimers appeared to alter the secondary and tertiary structure, but did not fragment the DNA or alter its primary structure. Complexed DNA was protected against degradation by either specific nucleases or cellular extracts containing nuclease activity.

Furthermore, the transfection ability of PAMAM dendrimers was found to be related to its acidic dissociation constant (23). Hui et al (26) investigated the ability of G5 PAMAM dendrimers to bind and transfer DNA to cells, and demonstrated that at $\mathrm{pH}$ 5.0, 7.0 and 9.0, PAMAM dendrimers were able to bind DNA, while at pH 3.0, PAMAM dendrimers had little ability to bind DNA. PAMAM dendrimer-DNA complexes $(4: 1, \mathrm{w} / \mathrm{w})$ protected DNA from digestion at $\mathrm{pH} 3.0$, 5.0, 7.0 and 9.0, as well as in the serum.

\section{Endeavors to enhance transfection efficiency}

Gene therapy is an efficient and economical approach to disease prevention and therapy. Efficient gene delivery and expression of exogenous genes in cells are the most important features. Although non-viral carriers are associated with various advantages including non-immunogenicity, low cytotoxicity and low cost, their low transfection efficiency compared to that of viral carriers limits their application in clinical gene therapy. In order to be utilized as a potential gene-transfer vector, improvement of the transfection efficiency of PAMAM dendrimers and expression of exogenous DNA in cultured 
cells or in vivo must be ensured. Endeavors to enhance the transfection efficiency have been explored, including the application of fractured dendrimers after heat treatment, dendrimer surfaces conjugated with oligopeptides, steroid or metal material, combination with the addition of cationic excipients, such as DEAE-dextran, and the emerging of new families. These may contribute to improvement of transfection activity.

Heat treatment. Tang et al (14) reported that further transfection efficiency was enhanced by the use of fractured (hydrolysisdegraded) dendrimers after heat treatment, since the transfection activity of PAMAM dendrimers is related to both the initial size of the dendrimer and its degree of degradation. After heat treatment in a variety of solvolytic solvents, including water and butanol, the dendrimers were significantly degraded, resulting in a heterodisperse population of compounds with molecular weights ranging from very low $(<1,500 \mathrm{Da})$ to several tens of kilodaltons. The compound facilitating transfection was found to be the high-molecular weight component of the degraded product and was denoted as a 'fractured' dendrimer. Fractured dendrimers exhibit an increased apparent volume change as measured by an increase in the reduced viscosity upon protonation of the terminal amines as $\mathrm{pH}$ is reduced from 10.5 to 7.2, whereas intact dendrimers do not exhibit this volume change. The transfection activity of the dendrimers was dramatically enhanced ( $>50$-fold).

Surface modification. Recently, diverse approaches have been undertaken to potentiate non-viral gene carriers, including linking or conjugating cell-specific ligands and TAT-derived peptides or oligopeptides, such as oligoarginine derivatives (27). Various basic peptides known as protein transduction domains or membrane translocalization signals have been identified and characterized and have been introduced in various therapeutic applications for the delivery of drugs, proteins, oligonucleotides and plasmid DNA. Note that these sequences usually contain positively charged amino acid residues, including arginine or lysine. Although it remains unclear whether entry into cell membranes follows an endocytic or non-endocytic pathway or by direct penetration into membranes, the phenomenon of enhanced transportation into cells has been reported in numerous studies (28-30).

PAMAM dendrimers modified with arginine oligopeptides were recently reported to show a high level of transfection efficacy. Choi et al designed a novel type of arginine-rich dendrimer, which conjugated with 1-arginine (PAMAM-Arg). This dendrimer demonstrated increased gene-delivery potency compared to native PAMAM dendrimers and PAMAM-Lys. The cytotoxicity and transfection efficiencies for 293, HepG2 and Neuro 2A cells were measured and compared to polyethylenimine (PEI) and PAMAM. In addition, transfection experiments were performed in primary rat vascular smooth muscle cells. PAMAM-Arg demonstrated greatly enhanced transfection efficiency, suggesting that the 1-arginine-grafted PAMAM dendrimer has the potential to be a novel genedelivery carrier for gene therapy (31). In 2006, Kim et al (32) investigated the transfection efficiency of PAMAM-Arg in primary cortical cultures compared to other gene carriers, such as native PAMAM, polyethylenimine (BPEI) and Lipofectamine; PAMAM-Arg/DNA complexes showed particularly high transfection efficiencies and low cytotoxicities in primary cortical cells. Efficient transfection was not limited to neurons, but was extended to all three glial cells (astrocytes, microglia and oligodendrocytes) that were present in these primary cortical cultures. Nam et al (17) designed and synthesized a new biodegradable polymer, e-PAM-R, which was modified with arginine by esterification. This polymer was expected to have two advantages for gene delivery: an enhanced transfection efficiency as a result of the introduction of arginine for DNA carriers, and fast biodegradation able to lower cytotoxicity and deliver antisense ODNs and small interfering RNAs to modulate gene expression. This suggested that the transfection efficiency of e-PAM-R G4 was higher than that of either G3 or G2, and was similar to PAMAM-R G4 with favorable cell viability (17).

With the exception of arginine and ornithine residues, phenylalanine-modified, $\alpha$ cyclodextrin or folate-conjugated dendrimers were found to show increased transfection activity, concomitant with an increasing number of terminal phenylalanine residues and lower cytotoxicity (33-35). Kono et al (36) designed PAMAM G4 dendrimers with hydrophobic amino acid residues, thus achieving an efficient transfection of cells through synergy of the proton sponge effect induced by the dendrimer tertiary amines and hydrophobic interaction by the amino acid residues. Transfection activity of these phenylalanine-modified dendrimers for CV1 cells, an African green monkey kidney cell line, increased concomitantly with the increasing number of terminal phenylalanine residues, apart from the dendrimer with 64 phenylalanine residues, which showed poor water solubility and barely formed a complex with DNA at neutral $\mathrm{pH}$.

The nuclear membrane is one of the main barriers in polymer-mediated intracellular gene delivery. One previous study reported that glucocorticoid receptors dilated the nuclear pore, resulting in translocation into the nucleus (37). Under this condition, the glucocorticoid dexamethasone was conjugated to a PAMAM dendrimer, and the effect of the conjugation was investigated by Choi et al (38). PAM-dexa/plasmid DNA complexes exhibited the highest transfection efficiency to 293 cells. This indicates that this technique with dexamethasone may be useful for gene delivery using polymeric gene carriers and for the development of efficient polymer vectors.

The blood-brain barrier (BBB) poses great difficulties for gene delivery to the brain. Most current gene vectors do not cross the BBB after intraveneous administration and must be administered via craniotomy or intracerebral injection, which are highly invasive and incapable of delivering exogenous genes to global areas of the brain. Huang et al (39) successfully synthesized PAMAM-PEG-Tf through the conjugation of $\mathrm{Tf}$ with PAMAM-PEG and greatly increased exogenous gene expression in the brain. This new vector demonstrated a concentration-dependent cellular uptake compared to PAMAM and PAMAM-PEG in vivo, as well as a much higher transfection efficiency. This suggests that PAMAM-PEG-Tf holds great promise for efficient, non-invasive, brain-targeting gene delivery.

Combination with other materials. Compounding PAMAM with cationic lipids may be beneficial to increase the level of transfection expression. Recently, cationic liposomes were approved for wide use in gene delivery. Cationic polypeptides, such as protamine sulfate and polylysine, are FDA-approved 
compounds with documented safety profiles that may be readily used as adjuvants in human gene-therapy protocols (40-42). Whether complexes of PAMAM and cationic liposomes enhance transfection efficiency has not yet been reported. Additional compounds, such as DEAE-dextran, appear to alter the nature of PAMAM-DNA complexes, and enhanced transfection efficiency was reported $(23,43)$. Electron microscopy revealed that plasmid DNA complexing to a highgeneration dendrimer (G10) formed large aggregates hundreds of angstroms in diameter. However, when the cells were treated with dimethyl sulfoxide to permeabilize their membranes, they did not demonstrate enhanced transfection efficiency with DNA-dendrimer complexes, suggesting that enhancement of transfection with DEAE-dextran may be due to an alteration in the DNA/dendrimer complex rather than in the cell membrane. Also, DEAE-dextran is a vehicle for gene delivery. With progressive increases in the dendrimer/DNA charge ratio, an increase in the amount of low-density, soluble complexes was observed. The ability of dendrimers to complex and form aggregates with DNA is crucial for efficient transfection and the function of the complexed DNA (44).

A novel PAMAM dendrimer. A new family of PAMAM dendrimers that uses a pentaerythritol derivative (PD) as a core possessing 12 branches was synthesized by Wang et al (45). The synthesized PD dendrimers of generations 3-5 efficiently condensed DNA into nanoscale complexes with slightly positive charges, and showed a higher transfection efficiency with a much lower cytotoxicity than the commercial non-viral gene carriers PEI, polylysine (PLL) and PAMAM dendrimers with an ethylenediamine core (generations 5 and 7). Patil et al (46) reported a novel, internally quaternized, surface-acetylated PAMAM generation 4 dendrimer (QPAMAM-NHAc). As a nanocarrier, it possessed the following two advantages: a modified neutral dendrimer surface resulting in low cytotoxicity and enhanced cellular internalization, and the existence of cationic charges inside the dendrimer resulting in highly organized compact nanoparticles which can potentially protect nucleic acids from degradation.

In conclusion, the novel gene carriers, PAMAM dendrimers, have been demonstrated to bind and protect DNA and are effectively delivered into cells both in vitro and in vivo. Their physicochemical properties, such as small size and markedly reduced cytotoxicity, make them promising substitutes for existing gene carriers in gene therapy. In the future, development of gene therapy with nanoparticle carriers may be beneficial to numerous patients with exogenous or endogenous sources of inner ear diseases.

\section{Acknowledgements}

This study was sponsored by the Special Program for Key Basic Research of the Ministry of Science and Technology, China (grant no. 2009CB526504), and the Natural Outstanding Youth Foundation of China (30925035).

\section{References}

1. Duan M, Venail F, Spencer N and Mezzina M: Treatment of peripheral sensorineural hearing loss: gene therapy. Gene Ther 11 (Suppl 1): 51-56, 2004.
2. Ge X, Jackson RL, Liu J, et al: Distribution of PLGA nanoparticles in chinchilla cochleae. Otolaryngol Head Neck Surg 137: 619-623, 2007.

3. Tamura T, Kita T, Nakagawa T, et al: Drug delivery to the cochlea using PLGA nanoparticles. Laryngoscope 115: 2000-2005, 2005.

4. Zou J, Saulnier P, Perrier T, et al: Distribution of lipid nanocapsules in different cochlear cell populations after round window membrane permeation. J Biomed Mater Res B Appl Biomater 87: 10-18, 2008.

5. Praetorius M, Brunner C, Lehnert B, et al: Transsynaptic delivery of nanoparticles to the central auditory nervous system. Acta Otolaryngol 127: 486-490, 2007.

6. Sun H, Jiang M and Zhu SH: In vitro and in vivo studies on hydroxyapatite nanoparticles as a novel vector for inner ear gene therapy. Zhonghua Er Bi Yan Hou Tou Jing Wai Ke Za Zhi 43: $51-57,2008$

7. Jiang M, Zhang YQ, He GX and Sun H: Protective effect of NT-3 gene mediated by hydroxyapatite nanoparticle on the cochlea of guinea pigs injured by excitotoxicity. Zhong Nan Da Xue Xue Bao Yi Xue Ban 32: 563-567, 2007.

8. Roy S, Johnston AH, Newman TA, et al: Cell-specific targeting in the mouse inner ear using nanoparticles conjugated with a neurotrophin-derived peptide ligand: potential tool for drug delivery. Int J Pharm 390: 214-224, 2010.

9. Praetorius M, Pfannenstiel S, Klingmann C, Baumann I, Plinkert PK and Staecker H: [Expression patterns of non-viral transfection with GFP in the organ of Corti in vitro and in vivo. Gene therapy of the inner ear with non-viral vectors]. HNO 56: 524-529, 2008 (In German).

10. Zhang Y, Liu JY, Yang F, et al: A new strategy for assembling multifunctional nanocomposites with iron oxide and aminoterminated PAMAM dendrimers. J Mater Sci Mater Med 20: 2433-2440, 2009.

11. Liu XX, Rocchi P, Qu FQ, et al: PAMAM dendrimers mediate siRNA delivery to target Hsp27 and produce potent antiproliferative effects on prostate cancer cells. ChemMedChem 4: 1302-1310, 2009

12. Navarro G and de Ilarduya CT: Activated and non-activated PAMAM dendrimers for gene delivery in vitro and in vivo. Nanomedicine 5: 287-297, 2009.

13. Tomalia DA, Baker H, Dewald J, Hall M, Kallos G, Martin S, Roeck J, Ryder J and Smith P: A new class of polymers: starburstdendritic macromolecules. Polymer J 17: 117, 1985.

14. Tang MX, Redemann CT and Szoka FC Jr: In vitro gene delivery by degraded polyamidoamine dendrimers. Bioconjug Chem 7 : 703-714, 1996.

15. Jansen JF, de Brabander-van den Berg EM and Meijer EW: Encapsulation of guest molecules into a dendritic box. Science 266: 1226-1229, 1994.

16. Roberts JC, Bhalgat MK and Zera RT: Preliminary biological evaluation of polyamidoamine (PAMAM) Starburst dendrimers. J Biomed Mater Res 30: 53-65, 1996.

17. Nam HY, Hahn HJ, Nam K, et al: Evaluation of generations 2, 3 and 4 arginine modified PAMAM dendrimers for gene delivery. Int J Pharm 363: 199-205, 2008.

18. Lee $\mathrm{H}$ and Larson RG: Multiscale modeling of dendrimers and their interactions with bilayers and polyelectrolytes. Molecules 14: 423-438, 2009

19. Landmark KJ, Dimaggio S, Ward J, et al: Synthesis, characterization, and in vitro testing of superparamagnetic iron oxide nanoparticles targeted using folic acid-conjugated dendrimers. ACS Nano 2: 773-783, 2008.

20. Tekade RK, Kumar PV and Jain NK: Dendrimers in oncology: an expanding horizon. Chem Rev 109: 49-87, 2009.

21. Klajnert B and Bryszewska M: Dendrimers: properties and applications. Acta Biochim Pol 48: 199-208, 2001.

22. Shakhbazau A, Isayenka I, Kartel N, et al: Transfection efficiencies of PAMAM dendrimers correlate inversely with their hydrophobicity. Int J Pharm 383: 228-235, 2010.

23. Haensler J and Szoka FC Jr: Polyamidoamine cascade polymers mediate efficient transfection of cells in culture. Bioconjug Chem 4: 372-379, 1993.

24. Kukowska-Latallo JF, Bielinska AU, Johnson J, Spindler R, Tomalia DA and Baker JR Jr: Efficient transfer of genetic material into mammalian cells using Starburst polyamidoamine dendrimers. Proc Natl Acad Sci USA 93: 4897-4902, 1996.

25. Bielinska AU, Kukowska-Latallo JF and Baker JR Jr: The interaction of plasmid DNA with polyamidoamine dendrimers: mechanism of complex formation and analysis of alterations induced in nuclease sensitivity and transcriptional activity of the complexed DNA. Biochim Biophys Acta 1353: 180-190, 1997. 
26. Hui Z, He ZG, Zheng L, Li GY, Shen SR and Li XL: Studies on polyamidoamine dendrimers as efficient gene delivery vector. J Biomater Appl 22: 527-544, 2008.

27. Tung $\mathrm{CH}$ and Weissleder R: Arginine containing peptides as delivery vectors. Adv Drug Deliv Rev 55: 281-294, 2003.

28. Futaki S: Arginine-rich peptides: potential for intracellular delivery of macromolecules and the mystery of the translocation mechanisms. Int J Pharm 245: 1-7, 2002.

29. Torchilin VP, Rammohan R, Weissig V and Levchenko TS TAT peptide on the surface of liposomes affords their efficient intracellular delivery even at low temperature and in the presence of metabolic inhibitors. Proc Natl Acad Sci USA 98: 8786-8791, 2001.

30. Suzuki T, Futaki S, Niwa M, Tanaka S, Ueda K and Sugiura Y: Possible existence of common internalization mechanisms among arginine-rich peptides. J Biol Chem 277: 2437-2443, 2002.

31. Choi JS, Nam K, Park JY, Kim JB, Lee JK and Park JS: Enhanced transfection efficiency of PAMAM dendrimer by surface modification with L-arginine. J Control Release 99: 445-456, 2004.

32. Kim JB, Choi JS, Nam K, Lee M, Park JS and Lee JK: Enhanced transfection of primary cortical cultures using arginine-grafted PAMAM dendrimer, PAMAM-Arg. J Control Release 114: 110-117, 2006

33. Kumar A, Yellepeddi VK, Davies GE, Strychar KB and Palakurthi S: Enhanced gene transfection efficiency by polyamidoamine (PAMAM) dendrimers modified with ornithine residues. Int J Pharm 392: 294-303, 2010.

34. Arima H: [Polyfection as nonviral gene transfer method - design of novel nonviral vector using alpha-cyclodextrin]. Yakugaku Zasshi 124: 451-464, 2004 (In Japanese).

35. Kang C, Yuan X, Li F, et al: Evaluation of folate-PAMAM for the delivery of antisense oligonucleotides to rat C6 glioma cells in vitro and in vivo. J Biomed Mater Res A 93: 585-594, 2010.

36. Kono K, Akiyama H, Takahashi T, Takagishi T and Harada A: Transfection activity of polyamidoamine dendrimers having hydrophobic amino acid residues in the periphery. Bioconjug Chem 16: 208-214, 2005.
37. Shahin V, Albermann L, Schillers H, et al: Steroids dilate nuclear pores imaged with atomic force microscopy. J Cell Physiol 202: 591-601, 2005.

38. Choi JS, Ko KS, Park JS, Kim YH, Kim SW and Lee M: Dexamethasone-conjugated poly(amidoamine) dendrimer as a gene carrier for efficient nuclear translocation. Int J Pharm 320: 171-178, 2006.

39. Huang RQ, Qu YH, Ke WL, Zhu JH, Pei YY and Jiang C: Efficient gene delivery targeted to the brain using a transferrinconjugated polyethyleneglycol-modified polyamidoamine dendrimer. FASEB J 21: 1117-1125, 2007.

40. Li S, Rizzo MA, Bhattacharya S and Huang L: Characterization of cationic lipid-protamine-DNA (LPD) complexes for intravenous gene delivery. Gene Ther 5: 930-937, 1998.

41. Tan Y, Whitmore M, Li S, Frederik P and Huang L: LPD nanoparticles - novel nonviral vector for efficient gene delivery. Methods Mol Med 69: 73-81, 2002.

42. Sorgi FL, Bhattacharya S and Huang L: Protamine sulfate enhances lipid-mediated gene transfer. Gene Ther 4: 961-968, 1997.

43. Malik N, Wiwattanapatapee R, Klopsch R, et al: Dendrimers: relationship between structure and biocompatibility in vitro, and preliminary studies on the biodistribution of 125I-labelled polyamidoamine dendrimers in vivo. J Control Release 65: 133-148, 2000.

44. Bielinska AU, Chen C, Johnson J and Baker JR Jr: DNA complexing with polyamidoamine dendrimers: implications for transfection. Bioconjug Chem 10: 843-850, 1999.

45. Wang Y, Kong W, Song Y, et al: Polyamidoamine dendrimers with a modified Pentaerythritol core having high efficiency and low cytotoxicity as gene carriers. Biomacromolecules 10: 617-622, 2009.

46. Patil ML, Zhang M, Betigeri S, Taratula $\mathrm{O}, \mathrm{He} \mathrm{H}$ and Minko $\mathrm{T}$ Surface-modified and internally cationic polyamidoamine dendrimers for efficient siRNA delivery. Bioconjug Chem 19: 1396-1403, 2008. 